\title{
Polymeric Dye Film Deposition in Vacuum
}

\author{
Kostyantyn Grytsenko ${ }^{1^{*}}$ and Yurii Slominskii ${ }^{2}$ \\ ${ }^{1}$ Institute of Semiconductor Physics, Laboratory of Semiconductor Molecular Systems, Ukraine
}

${ }^{2}$ Institute of Organic Chemistry, Department of Color and Structure of Organic Compounds, Murmanska, Ukraine

Received: October 26, 2017; Accepted: November 8, 2017; Published: November 10, 2017

*Corresponding author: Kostyantyn Grytsenko, Institute of Semiconductor Physics, Laboratory of Semiconductor Molecular Systems, Ukraine. Tel: +38 044 5255530; Fax: +38 044 5255530; E-mail: d.grytsenko@gmail.com

\begin{abstract}
A new type of evaporable organic molecules containing unsaturated reactive fragment has been developed for vacuum deposition of the polymer dye film. The designed molecules contain the core chromophore for an active optoelectronic function. The properties of the thin solid films, produced using new evaporationactivation-polymerization method from the novel compounds possess enhanced smoothness, hardness and thermal stability. Some dyes form nano-wires by a self-assembly between the two neighbour gold strips on the silicone surface.
\end{abstract}

Keywords: Dye; Vacuum; Evaporation; Activation; Polymerization; Thin Film; Optical Spectra; Annealing

\section{Introduction}

The development of organic electronics has started at the end of 20th century. Now the world market of devices based on functional organic compoundshas been estimated at billions of dollars. Organic light emitting devices, organic field effect transistors, various optical and electrical sensors represent just a short list of the industrial applications of the organic functional materials. The requirements to organic material vary depending on the application, but the long term of usage is necessary. Functional organic layer can be formed from molecules of two types: a small molecule (dye) or a macromolecule (polymer). The polymer materials are more robust and have a longer lefe time. While the polymer film can be deposited by wet method only with a few exceptions, the dye layer can be made by both wet methods and vacuum deposition technology. Only some homopolymers can be deposited by heating, decomposition and secondary polymerization in vacuum [1]. Widely used in the inorganic electronics vacuum deposition is not very compatible with wet methods for in-line production. Moreover, wet methods have some restrictions for production of multi-layered and multi-component systems. Using vacuum deposition, there are no restrictions in wettability of components and layers. The production of the polymer film by the gas phase deposition provides the benefit of ease gas processing (small volatile molecules) and stability of the polymer material. The most vivid example is the di-para- xylylene gas phase pyrolytic activation-polymerization without by-products release. The obtained polyparaxylylene films are characterized with the high thermal and chemical stability and they have found applications in various industrial products. Produced by deposition in vacuum, organic solids frequently appear in the form of mesh, needles,discontinuous aggregates. The stability of such "films" is not satisfying. It can be improved by increase of compound molecular weight, but this leads to lose of evaporability. Therefore, to find a solution to the problem of producing stable and smooth polymeric films from organic chromophore by deposition in vacuum is very important for industrial applications. Previously attempts to polymerize dyes in plasma were made in Japan, using standard molecules of phthalocyanine andmerocyanine[2-4]. The deposits were stable polymers, but their optical absorption was reduced. Authors emphasized, that the part of the molecules were decomposed. Later the plasma-assisted dye deposition was performed [5]. Dye molecules were sublimed and introduced to the already glowing discharge in benzene vapor. Authors reported, that the part of the dye molecules was damaged. Thus, more dyes were "polymerized" using plasma with an aim to produce stable films[6-9]. From IR-spectra authors concluded, that the dye molecules were incorporated in the "polymer" film, but no another compound, except dye, was in the plasma. It means, that the part of the molecules was decomposed to produce "polymer" matrix.

In general, the energetic species of low temperature plasma destruct chemical bonds in the molecule in the gas phase. Then molecules with opened bonds are condensing on the substrate surface and interacting with each other, producing a "polymer" film. When the classic plasma treats a molecule with the size larger, thanthe small monomer (namely chromophore), it leads to destruction of themolecule in a random way with loss of the chromophore optical properties. Moreover, to ignite the plasma, it is necessary to produce a conductive path in the gas, which leads to ionization and decomposition of the part of the molecules. Therefore, both classic plasma polymerization and standard chromophores are not suitable for production of the polymer film with optoelectronic properties of the pristine molecules.But 
the attempt to produce polymer films from organic compounds in plasma is still in progress[10,11], indicating the importance of the stable organic functional thin films.

Thus, the new type of the chromophore containing unsaturated group, which is able to open only after evaporation but before the condensation has to be designed. The new technology for selective activation of this unsaturated bond in the gas phase should be created.

\section{Experimental Details}

Deposition of the thin dye films have been performed using UVN74 vacuum installation. Base pressure in the chamber was 10-3 Pa. Glass and Si plates have been used for film deposition. Gold film was deposited on part of the glass and Si plates by thermal evaporation. Dye powder have been heated in quartz crucible. The vapor was activated in the gas phase. Growth rate and film thickness were measured with Sigma quartz thickness monitor. Film thickness, morphology and nanohardness were studied using atomic force microscope (AFM) NanoscopelIIa Dimension $3000 \mathrm{TM}$ at room temperature in a tapping mode. Optical spectra were measured during film heating in the air using Polytec spectrometer.

\section{Results and Discussion}

Creation of comparatively large evaporable and reactive organic molecule needed special design. The molecule has to have a special groupthat is not able to react with a neighboring group during heating up to the evaporation temperature. This bond has to be opened by activation in the gas phase only, thus supplying active diradicalsto the substrate surface. They has to be condensed at room temperature on the substrate surface, interact with each other to produce a polymeric solid. It was was hardly possible to calculate the structure of the necessary molecule due to close values of the energies $(\mathrm{E})$ of the all $\mathrm{C}=\mathrm{C}$ bonds. The molecule have to satisfy the following rule:

Eevaporation $<$ Eopening unsaturated bond $<$ Eall other bonds in the molecule

The energy of activation has to be matched with the energy of the unsaturated bond opening.Following this approach, less energetic plasma was tested for polymerization of several comparatively large monomers with unsaturated bond by Grytsenko et al. [12]. It was found that decrease of the discharge power in the monomeric gaseous medium led to the formation of the solid film with the properties close to classic polymer. Stable films of a small merocyanine dye with optical absorption in ultraviolet region were also deposited in plasma. Later various molecules were designed and tested. Ultimately the desired compounds were designed and synthesized [13].

Depending on the molecule structure, activation conditions and substrate material, the solids with different morphologies were produced.Experimental results, obtained using two reactive merocyanine compounds, 1 and 2, are presented in this paper.

Morphology and nano-mechanical properties of the films were varied by changing activation parameters (Figure 1,2). The films, deposited with the best activation conditions, were smooth at nano-level and showed enhanced nano-hardness. The roughness of the film, deposited with activation by small power classic low temperature plasma was $18 \mathrm{~nm}$, while the roughness of the film from the same dye but deposited with the new activation method was 1,4 nm, (Figure 1). Several dyes have revealed nanowire growth by self-assembly. Figure 1 shows the nanowires (about 30 micrometers , 1 micrometers) lying on the Si surface with both ends attached to the side surfaces of the two neighbor gold strips. Usually the second end of the nanowire is loose [14,15].The additional efforts were necessary to connect the loose end with another surface. The optimal activation conditions were strictly individual for each dye.
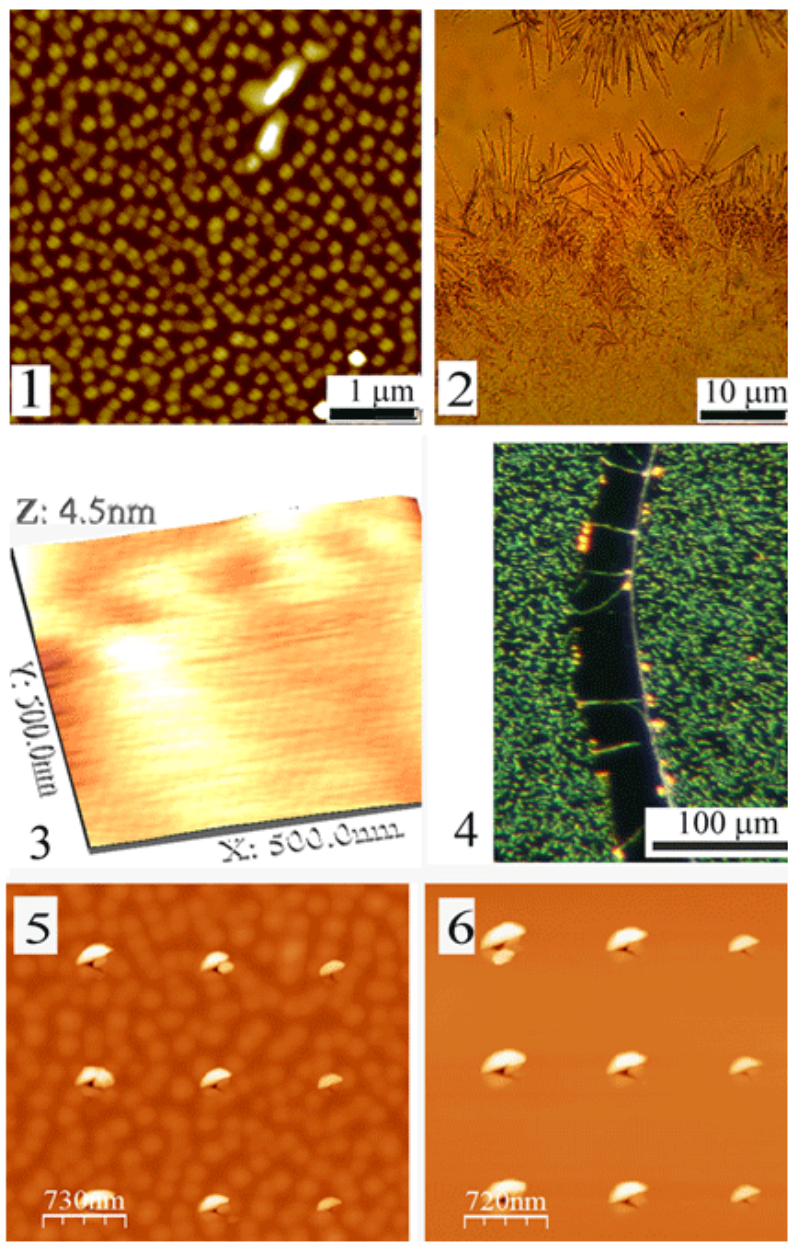

Figure 1: Morphology of the 1 compound deposits on glass, produced with various $(1,2,3)$ activation conditions, $3-3$ D image of the surface of the film; 4 - nanowires from compound 2 on Si between two gold strips; 5 - film of compound 1 deposited with low power plasma, 6 - film of compound 1 deposited with new activation method; marks were made with AFM tip during hardness measurements. 


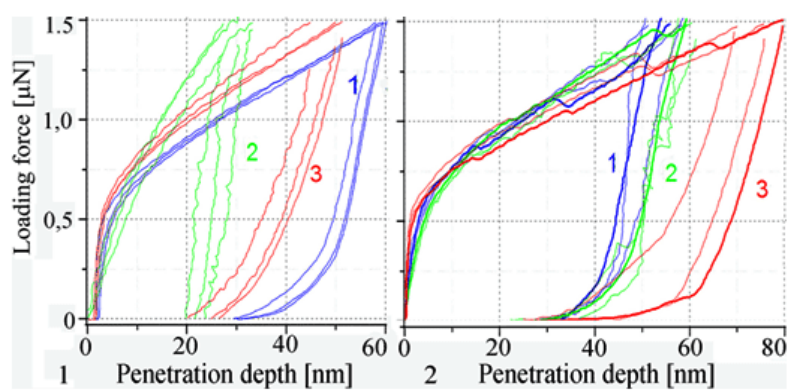

Figure 2: Load-penetration curves of the films, deposited with different activation methods: dyes 1 and 2; 1,2,3 - activation conditions.

Optical spectra of the films deposited with activation contained one monomeric band of the pristine chromophore, while the films of the same dyes but deposited without activation contained broadened and red-shifted band. The films, deposited with the best activation conditions, revealed no transformations of the optical spectra during heating in air to decomposition temperature (Figure 3). The decomposition temperature for the films, deposited with activation was higher, than for the films, deposited by the classic thermal evaporation. Moreover, the decomposition temperature of the films exceeded the evaporation temperatureof the pristine dyes. The enhanced properties of the films can be explained by the formation of the polymeric (oligomeric) material. This development is just the first stage of the research: several types of the much bigger compounds for polymerization in vacuum, previously unknown, were designed. The technologies for evaporation-activation-polymerization of these new reactive chromophores were presently developed and tested. The second set of the new molecules containing two active functionalities was designed, in particular, evaporable organic salts, containing unsaturated group. After their activation upon evaporation, thin films of cationic dye polymer were obtained. The molecules, containing core chromophore for photovoltaic, the unsaturated group and the group for bonding with gold will be synthesized. The compounds with reactive groups open the pathways to form a soft matter with enhanced properties, for example, co-polymerization of two insoluble dyes to produce one phase solid with no phase separation during heating and so on. The developed compounds and the deposition technology open the new direction for creation stable active elements for various optoelectronic devices on nano- and macro-level.
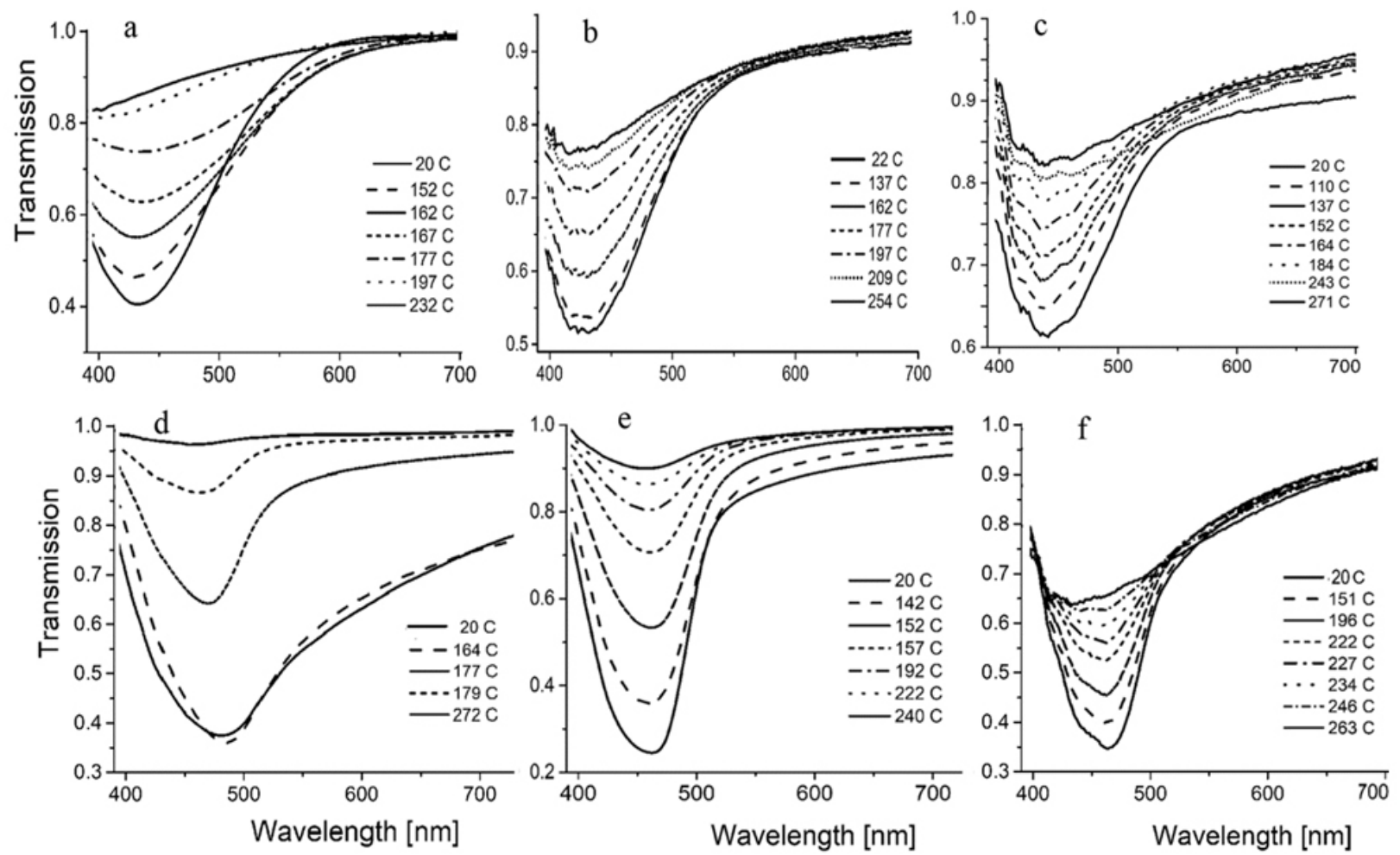

Figure 3: Optical transmission spectra of the films of the dye 1 (a-c) and 2 (d-f) during heating in air: a,d - films deposited by conventional thermal evaporation, b,c,e,f - - films deposited by activation methods 2 and 3. 


\section{Conclusions}

The developed evaporable organic compounds with the reactive group in combination with the activation methods for selective reaction of this group during the deposition process lead to the formation of smooth and highly stable thin organic films. Variations in the activation parameters allow controlled morphology of the deposited films from the smooth surface at nano-level to the self-assembled nano-wires in multilayered nanostructured system.

\section{References}

1. Gritsenko K P, Krasovsky A M. Thin film deposition of polymers by vacuum degradation. Chemical Reviews. 2003;103(9):3607-3650. doi: $10.1021 /$ cr010449q

2. Kashiwazaki N. Iodized Polymeric Yb-Diphthalocyanine Films Prepared by Plasma Polymerization Method. Jap. J. Appl. 1992;31(1) 1892.

3. Inagaki N, Tasaka S, Ikeda Y. Plasma polymerization of copper phthalocyanine and application of the plasma polymer films to $\mathrm{NO}_{2}$ gas sensor device. J. Appl. Polymer Sci. 1995; 55(10):1451-1464.

4. Inoue M, Morita H, Takai Y, Mizutani T, Ieda M. Photovoltaic Cells of Merocyanine Dye Polymer Thin Films Prepared by Plasma Polymerization Method. Jap. J. Appl. Phys. 1988;27:1059.

5. Homilius F, Heilmann A, von Borzyskowski C. Plasma polymer thin films with encapsulated dye molecules and silver nanoparticles. Surface \& Coat. Technol. 1995;74-75:594-597.

6. Barranco, Groening P. Fluorescent Plasma Nanocomposite Thin Films Containing Nonaggregated Rhodamine 6G Laser Dye Molecules,Langmuir. 2006;22(16):6719-6722. doi: 10.1021/ la053304d
7. Barranco, Aparicio F, Yanguas-Gil A, Groening P, Cotrino J, GonzálezElipe A R. Optically Active Thin Films Deposited by Plasma Polymerization of Dye Molecules. Chem. Vap. Deposition. 2007;13(67):319-325.

8. Blaszczyk-Lezak I, Aparicio F J, Borras A, Barranco A, Alvarez-Herrero A, Fernandez-Rodriguez $M$, et al. Optically Active Luminescent Perylene Thin Films Deposited by Plasma Polymerization. J. Phys. Chem. C . 2009;113(1):431-438.

9. Aparicio F J, Borras A, Blaszczyk-Lezak I, Groening P, AlvarezHerrero A, Fernandez-Rodriguez M, et al. Luminescent and Optical Properties of Nanocomposite Thin Films Deposited by Remote Plasma Polymerization of Rhodamine 6G. Plasma Process. Polym. 2009; 6(1):17-26.

10. Palacios J C, Olayo M G, GuillermoJ Cruz, J. A. Chávez-Carvayar. Meyer-Ne ldelRuleinPlasmaPolythiopheneThinFilms, OpenJ. PolymerChemistry. 2014;4(3):31-37. doi: 10.4236/ojpchem.2014.43004

11. Martín M A. Remote plasma assisted fabrication of functional organic and hybrid thin films and supported nanostructures. Ph.D. Thesis, Universidad de Sevilla. 2015.

12. Gritsenko K P, Tolstopyatov E M, Yagupolskii L M, Yagupolskii Yu L, Kremlev M M, Slominsky Yu Let al. Plasma polymerisation of complex organic molecules, Conference Ionizing Radiation and Polymers-98. Weinbohla.1998.

13. Tolstopyatov E, Grytsenko K, Kolomzarov Yu, Lytvyn P, Navozenko O, Schrader S, et al .Polymeric dye by gas phase deposition, Conference Polymer composites and tribology. Gomel, Belarus. 2013.

14. Zhao Y S, Zhan P, Kim J, Sun C, Huang J. Patterned Growth of Vertically Aligned Organic Nanowire Waveguide Arrays. ACS NANO. 2010; 4(3):1630-1636. doi: 10.1021/nn901567z

15. Tavares L, Kjelstrup-Hansen J, Thilsing-Hansen K, Rubahn H G. Organic nanofibers integrated by transfer technique in field-effect transistor devices. Nanoscale Research Lett. 2011;6: 319. 